\title{
Acoustic investigation of cavitation noise from offset ink film splitting
}

Joakim Voltaire and Andrew Fogden, Institute for Surface Chemistry, YKl, Stockholm, Vincent Craig, Australian National University, Daniel Jansson, Högskolan Dalarna, Falun, Niclas Jacobsson, Billerud AB, Skärblacka, Sweden

KEYWORDS: Film splitting, Printing, Ink tack, Cavitation noise

SUMMARY: The acoustic signal from the nip during film splitting between inked rollers and on ink transfer to paper was investigated on laboratory scale, using two sheet-fed offset inks (based on mineral oil and vegetable oil) and two coated fine papers (glossy and matte). Film splitting emits a broad noiselike acoustic spectrum in the range $5-50 \mathrm{kHz}$, from which the two simplest measures of average power and average frequency were derived. Using these two measures, four characteristic regimes of film splitting on the rollers could be distinguished as a function of ink load. Moreover, for intermediate amounts, average power was found to be accurately predicted by a simplistic model of sound produced by cavity expansion due to the under-pressure in the nip exit, thus providing a physical interpretation of the nip noise emission. For printing, the average power was higher for the glossy paper than the matte, presumably due to a more efficient sealing of the nip, or onset of ink setting. The vegetable oil-based ink gave higher power than its mineral oil counterpart. These results were found to be consistent with longer-time measurements of tack evolution from the Ink Surface Interaction Tester. This correlation and the verified theory thus provide support to practical applications of acoustic emission as an on-press monitoring tool.

\section{ADDRESSES OF THE AUTHORS: JoakimVoltaire and}

Andrew Fogden*: Institute for Surface Chemistry, YKI, Drottning Kristinas väg 45, SE-114 86 Stockholm, Sweden. Vincent Craig: Australian National University, Dept. of Applied Mathematics, Canberra ACT 0200, Australia.

Daniel Jansson: Högskolan Dalarna, SE-791 88 Falun,

Sweden. Niclas Jacobsson: Billerud Skärblacka AB, SE-617 10

Skärblacka, Sweden. *Current address, Andrew Fogden:

Australian National University, Dept. of Applied Mathematics, Canberra ACT 0200, Australia.

Corresponding author: Joakim Voltaire

(joakim.voltaire@surfchem.kth.se)

Splitting and transfer of ink films between roller and cylinder nips in offset printing is critical to both print quality and press runnability. The nip's narrow converging and diverging gaps result in a rapidly increasing pressure followed towards the exit by a subambient tensile stress or "tack" region, before the film splits (Patel and Dealy 1987). Splitting and tack are affected by cavitation, i.e. disruption of the otherwise continuous ink phase by the presence of gas or vapour bubbles. This study focuses on the sound generated by splitting and its relation to cavitation.

The study of film separation and tack dates back to Stefan's squeeze film experiments on liquids between parallel plates (e.g. Bikerman 1968), from which Reynolds developed a film lubrication theory which is the basis for coating flow computations. However, these are not valid in the film splitting and cavitation region that involves free boundaries and discontinuities. Sjodahl (1951) showed that film splitting produces filaments at the nip exit due to sub-ambient cavitation, with the deviation from Stefan's law explained by Banks and Mill (1953). Myers et al. (1958) later observed, using a high speed camera, cavitation at a critical speed for model dispersions. At the inception of cavitation, the reversed flow due to suction of fluid into the formed cavities was also visualised. At higher speed the number of cavities increased and dominated the separation. Voet and Geffken (1951) showed the importance of viscoelasticity for filament formation, in particular at high speed printing. Taylor and Zettlemoyer (1958) suggested that shear thinning and higher temperature in the nip reduced the cohesive strength in favour of cavitation. Dowson and Taylor (1979) defined two types of cavitation: gaseous cavitation in which gas from the surrounds entrains the liquid or releases from dissolved gas, and vaporous cavitation when the liquid pressure becomes lower than the vapour pressure of some component and causes its boiling. Ozogan and Young (1981) studied mineral oil and inks utilising the heat generated by cavitation, discovered by Coveney (1978). For the transparent mineral oil, the onset of cavitation on increasing speed involved formation of a bubble row and a small increase in temperature to a stable level. At higher speed the temperature increased to a new stable level with air fingers and travelling bubble clouds observed. The opaque inks exhibited the same temperature steps, but with an oscillating behaviour in the first step indicating that bubbles passed from stable to transient behaviour. Later studies addressed cavitation nucleation at paper surface asperities (De Grace and Mangin 1984) and air entrainment at the nip entrance (Bery and Loel 1992).

De Grace et al. (1992) investigated filament elongation in a print nip, and found that filaments broke further from the nip at higher ink loads and closer to the nip at higher speeds. Increasing speed also broadens the filament distribution (Glatter and Bousfield 1996), (Thomson and Young 1975). Recently, Ercan (2001) photographed filaments in a laboratory inking unit nip and filament residues from a laboratory print tester and compared to rheological and cavitation tensile tests on different liquids and inks, showing that filament average volume and size distribution increased with film thickness and print speed. The ink rheology, filamentation and cavitation scaled in a complicated manner. Besides experimental studies, much computation has been performed using elasto-hydrodynamic models for coating and printing nips, see e.g. (Dowson and Higginsson 1966), (Coyle 1988), (MacPhee et al. 1992), (Carvalho and Scriven 1996). A special concern has been 
the mechanism of "ribbing" instability at the meniscus, in which a sinusoidal pattern appears on the film surface. Ribbing occurs if the pressure gradient in the meniscus region caused by either viscous or inertial forces is too high (Pearson 1960). Computations involving the nonNewtonian property of the ink were carried out by Lim et al.(1996). Recently, Cioc (2004) presented an algorithm accounting for discontinuities and free boundaries involved in cavitation of bearings, which previously needed to be treated separately from the lubrication regime.

Noise emission and damage from transient cavitation are well-studied phenomena, see e.g. (Blake 1986), (Ceccio 1990), (Brennen 2005). Bubble dynamics can be described by the Rayleigh-Plesset equation. Transient collapsing cavities may in the far field be treated as single sources, where the inertia of the bubble collapse dominates. The hissing-crackling noise emission generated in a print nip is a manifestation of cavitation. Thus acoustic emission (AE) measurement techniques (Holroyd 1997) would, together with theory, be expected to provide insight into the mechanisms occurring, and a valuable complement to the above-mentioned techniques. Although AE has principally been used for detection of vibrations (e.g. of the press itself) or fracture in materials (e.g. the paper itself), one research group in Japan has though investigated film splitting noise from offset printing nips. Hayashi and Amari (1992) and Hayashi et al. (1993) showed that an increasing fountain solution level decreased the acoustic power, along with ink tack and filament length. Amari (1994) found that a varnish with a chemical gelling bond structure gave louder film splitting than one with physical bonds and lower tack. The power spectrum originating from the film splitting showed a broad band distribution between $10-30 \mathrm{kHz}$, with its highest intensity in the range $10-20 \mathrm{kHz}$. The sound emitted by paper release in a sheet-fed offset pilot press was studied by Iwasaki (1993), but not related to the film splitting mechanism. The acoustic method for probing film splitting phenomena was recently reinvestigated (Voltaire 2004) and applied to monitoring of a heat-set web offset press (Voltaire et al. 2004), where it was shown capable of detecting changes in film splitting in response to press and material conditions (ink and fountain solution feed, and ink and paper composition).

In the current study the film splitting sound in a laboratory inking and printing unit was measured, investigating the effect of ink amount, sheet-fed offset ink type and coated fine paper type, essentially in terms of the average power and average frequency from the broad band noise signal. A simplistic model was developed to explain dependence of average power on ink amount. This combination of experiment and theory will help in furthering the development of the acoustic method for on-line monitoring of offset printing.

\section{Materials and Methods}

\section{Materials and printing devices}

The two inks used were commercial cyan sheet-fed offset inks, Toplith and Ecolith (Sun Chemical), with their vehicle based on mineral and vegetable oil, respectively. The two papers were a matte and glossy commercial grade of coated fine paper $\left(115 \mathrm{~g} / \mathrm{m}^{2}\right)$, of identical composition, only distinguished by the degree of calendering. The $75^{\circ}$ sheet gloss, measured with a ZGM 1022 glossmeter (Zehntner $\mathrm{GmbH}$ Testing Instruments) as the average at five locations, is 80 and $17 \%$ for the glossy and matte papers, respectively. Their roughness was determined by a white light interferometric profilometer (New View 5010, ZYGO Corp.) over image areas of $3.4 \times 3.4 \mathrm{~mm}$, and its root mean square (rms) components for band-passed lateral length scales ranging from micro- to macro-scale are summarised in Table 1.

Table 1. Roughness distributions of the glossy and matte coated papers from white light profilometry.

\begin{tabular}{lcc}
\hline Wavelength interval $(\mu \mathrm{m})$ & \multicolumn{2}{c}{ Roughness, rms $(\mu \mathrm{m})$} \\
& Glossy & Matte \\
\hline $5-10$ & $0.099 \pm 0.001$ & $0.300 \pm 0.004$ \\
$10-20$ & $0.080 \pm 0.001$ & $0.241 \pm 0.009$ \\
$20-50$ & $0.162 \pm 0.009$ & $0.309 \pm 0.007$ \\
$50-100$ & $0.239 \pm 0.006$ & $0.462 \pm 0.009$ \\
$100-900$ & $0.623 \pm 0.030$ & $1.250 \pm 0.195$ \\
\hline
\end{tabular}

The inking unit (IGT AE, IGT Testing Systems) used in the experiments (Fig 1 a) consists of a large aluminium cylinder at the front, that moves sideways, a small motordriven aluminium cylinder at the back, and a polyurethane-coated top roller. The surface speed of the rollers is $0.17 \mathrm{~m} / \mathrm{s}$. A microphone was clamped in close proximity to the nip, central and perpendicular to its cylinder axis. Pre-weighed amounts of the inks, varied in the broad range from 0.5 to $100 \mathrm{~g} / \mathrm{m}^{2}$, were evenly applied to the top roller and subsequently distributed over the full width of the roller and cylinders. On attainment of an even distribution, the acoustic signal at the nip exit was monitored over a period of $1 \mathrm{~s}$. Several recordings were made over a longer duration (1 min.) to verify that the sound pressure level was stable within limits. The IGT inker motor was then switched off, and the time to selfstop of rotation was detected from a longer recording (12 $\mathrm{s})$. This time was interpreted as an indirect measure of the ink tack, expected to drag the free rotation of the cylinder. The same procedure was repeated after adding more ink to the roller.

A pre-determined amount of the ink, distributed on the rollers of the IGT unit, was uniformly transferred to a smaller roller, or print disc, then mounted on the laboratory printing unit (see Fig $1 b$ ). In particular, the printing

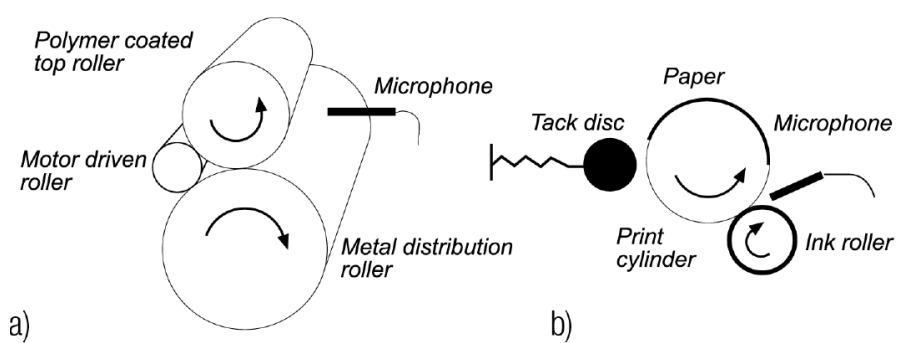

Fig 1. Principle illustration of acoustic monitoring of film splitting on a) ink distributor unit and b) printing and pull-off testing instrument. The former also possesses an extra distribution roller at the back (not shown), and a small print roller that is inked and then mounted on the printing unit. 
unit on the Ink Surface Interaction Tester (ISIT, Segan Ltd) was used for this purpose (Gane and Seyler 1994). The ink on the print disc is applied during a single rotation to a paper strip mounted using double-sided tape on the circumference of a larger cylinder. The nip pressure was set to $800 \mathrm{~N}$, the printing speed to $0.5 \mathrm{~m} / \mathrm{s}$, and the transferred ink amount stepped from 1 up to $5 \mathrm{~g} / \mathrm{m}^{2}$. The microphone was mounted close to the nip between print disc and paper-bearing cylinder, to record the sound during the ink splitting and transfer. In separate experiments, not involving acoustic measurement, the ISIT was operated in its standard mode in order to measure, at preset time intervals after printing, the "tack" force necessary to separate a rubber tack disc placed in contact with the printed area.

\section{Acoustic technique}

The principle of the acoustic measurement is illustrated in Fig 2. The microphone comprised a high level 1/4-inch condenser microcapsule of type 40BE (G.R.A.S ${ }^{\circledR}$ ), with its preamplifier connected to a power supply. Digital sampling was performed by a computer coupled to an external analogue digital converter (ADC, Data Translation $\left({ }^{\circledR}\right)$. The detectable frequency range was 10 $\mathrm{Hz}-50 \mathrm{kHz}$, with the upper limit set by the sampling rate of $100 \mathrm{kHz}$, and a sound pressure level (SPL) range of 40-168 dB relative to the $20 \mu \mathrm{Pa}$ audible limit. Using LabView ${ }^{\circledR}$ and DTLV-Link ${ }^{\circledR}$, the signal was sampled at a rate of $100 \mathrm{kHz}$ into buffers of $0.1 \mathrm{~s}$ that were concatenated into larger recordings. The raw data, in V, was converted back to pressure $(\mathrm{Pa})$ from the microphone's known sensitivity of $3.92 \mathrm{mV} / \mathrm{Pa}$.

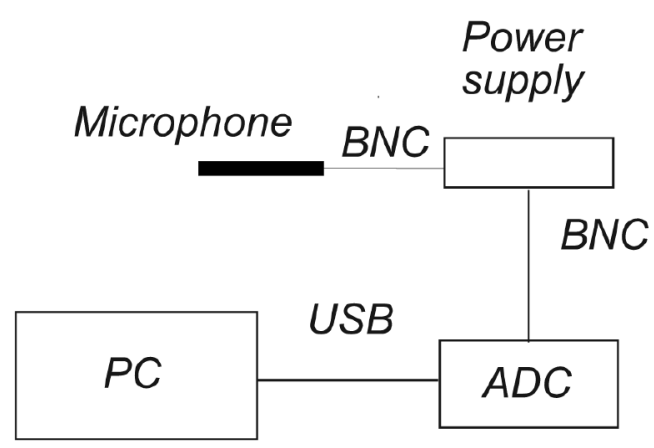

Fig 2. Flow chart of the acoustic measurement system.

\section{Signal processing}

Microphones measure sound pressure $p$ resulting from a local variation in both density and pressure, and hence a volumetric strain (Fahy 2003 p.27) . Considering a body in air with an equilibrium volume $V_{0}$ changing by amount $\delta V$, its strain is essentially an adiabatic and elastic process obeying Hooke's law:

$$
p=p_{t o t}-p_{0}=-c^{2} \rho_{0} \frac{\delta V}{V_{0}}=-\frac{1}{C} \frac{\delta V}{V_{0}}
$$

where $p_{\text {tot }}$ is the total pressure, $p_{0}$ the static pressure, $c$ the speed of sound, and $\rho_{0}$ and $C$ the equilibrium density and compressibility (or inverse bulk modulus) of the body, respectively. A sound wave is generated if a force accele- rates molecules locally so that this volume strain differs with time and position. The total mechanical energy, $E$, or "sound energy density", which unlike the sound pressure is a conserved quantity in the absence of dissipative processes, is given by the sum of kinetic $(K)$ and potential $(U)$ energy according to (Fahy 2003 p.76):

$$
E=K+U=\frac{\rho_{0} u^{2}}{2}+\frac{p^{2}}{2 \rho_{0} c^{2}}
$$

where $u$ is the net particle velocity travelling with the sound. As the microphone measures $p$, it will hence give an estimate of the potential energy of the sound. By a common convention (Oppenheim and Willsky 1997 p.6), a squared quantity has the unit of energy, which here is roughly motivated by $E q 2$. The sum of all squares in a finite sequence divided by the number of points $\mathrm{N}$ in the sequence is the mean square or the average power:

$$
\boldsymbol{P}_{a}=\frac{1}{N} \sum_{k=0}^{N-1} p_{k}^{2}=\Delta f \sum_{n=0}^{N / 2}{P S S D_{n}}
$$

The second equality is Parseval's theorem (Press et al. 1992 pp.504, 551), stating that the power in the time domain is equal to its representation in the frequency domain. Here $\Delta f$ is the frequency bin and PSD is the onesided power spectral density (power normalised to $1 \mathrm{~Hz}$ bandwidth) defined according to (Press et al. 1992 p.504):

$$
\begin{aligned}
& S_{n}=\frac{2}{N^{2}}\left|X_{n}\right|^{2} \quad 1 \leq n \leq \frac{N}{2}-1 \\
& P S D_{n}=\frac{S_{n}}{\Delta f}=\frac{N S_{n}}{f_{s}}
\end{aligned}
$$

where $S_{n}$ is the one-sided power spectrum, $f_{s}$ the sampling rate (here $100 \mathrm{kHz}$ ) and $X$ the discrete Fourier transform (DFT) calculated by the Fast Fourier Transform (FFT) (Press et al. 1992 p.504-510). For the endpoint frequencies, $n=0$ (i.e. corresponding to DC) and N/2 (the socalled Nyquist frequency), the power spectrum definition in $E q 4$ is halved. Different conventions exist, however the $P S D$ in $E q 4$ maintains the magnitude of the spectrum for any value of $N$.

White noise in the $P S D$ was reduced by the Welch method (Welch 1967), dividing the signal into a number (e.g. 10) of $50 \%$ overlapping sub-signals, multiplying each sub-signal with a Hann-window to smooth the discontinuous cut at the end points, calculating the PSD for each, and averaging all PSD's to one spectrum. From the spectral analysis (see below) it was clear that a highpass frequency-selective filter should be applied at $5 \mathrm{kHz}$ to exclude low frequency machinery noise and $1 / f$ noise (Smith 1997b p.172), and to enhance the broad band ink splitting sound. Average pressure is converted to sound pressure level $(S P L)$ in $\mathrm{dB}$ unit as follows:

$$
S P L(d B)=20 \log _{10} \frac{p_{r m s}}{2 \cdot 10^{-5}}=10 \log _{10} \frac{P_{a}}{4 \cdot 10^{-10}}
$$

where $p_{r m s}$ is the root mean square sound pressure. The 
spectral distributions were analysed both visually and quantitatively from an average frequency $f_{\mathrm{a}}$ here defined as the expectation value:

$$
f_{a}=\sum_{n=0}^{N / 2} P S D_{n} n \Delta f / \sum_{n=0}^{N / 2} P S D_{n}
$$

According to the Nyquist and Shannon rule (Press et al. 1992 p.500), the sampling rate has to be at least twice the sampled frequency band in order for it to be accurately reconstructed. Otherwise, frequency content that is higher than half the sampling rate (Nyquist frequency) will fold back as false aliases in the studied frequency domain. Thus if the analogue signal is not low pass filtered before being digitised, the obtained spectrum after $D F T$ will be contaminated and there is usually no cure for this. Alternatively, one may assume that higher frequencies are of minor importance, which can be justified if the $P S D$ appears to decrease in the high frequency range. In this work, no anti-aliasing filter was used, although the aliasing is expected to be considerable. For the purpose of relative measurements of total signal power above a specific frequency, say $5 \mathrm{kHz}$, this is not a serious matter, as most of that power (except for a minor noise fraction) comes from the film splitting, although it may have its real origin above $50 \mathrm{kHz}$. The average frequency may however be more severely affected by the aliasing.

\section{Theory}

To link the average power in $E q 3$ to the sound source, a (simplified) model for the roller nip noise, generated by the volume expansion of cavities after the nip centre, is required. A similar and well-studied phenomenon is the noise generated from travelling bubbles, e.g. around propellers and turbines under water (Brennen 2005). A gas/vapour bubble immersed into a liquid of higher density can, due to its higher compressibility, easily change its volume in reaction to the fluid pressure (see $E q$ 1). If the liquid pressure is lower than the vapour pressure in the bubble, the bubble will grow, counteracted by surface tension. It may then stabilize and oscillate at its natural frequency, though often non-linearly. If the bubble moves to a region of higher fluid pressure, it may rebound if possible or collapse (implode) while emitting noise to its surroundings (Young 1999 pp.115, 196, 253). The emitted noise is essentially due to the inertia and the volume acceleration of the bubble (Brennen 2005 p.142). In a print nip, cavities are nucleated, or expanded from already present nuclei, as a reaction to the tensile stress. However, the collapse mechanism differs from a submerged case, in that the compensation is made by a second fluid. If the rollers were immersed, the cavities would have collapsed, or stabilised in the ink phase outside the roller nip. The sound source in a print nip consists of a multitude of cavities located in a row along the cylinder axis, and consequently interference of these sources is expected. It is worth noting that the interaction of a cavity with a free surface (meniscus) can be complicated (Blake and Gibson 1987), including turbu- lence and scattering (Williams 1969), and that real cavities are neither spherical nor mono-sized and coalescence and bubble cloud formation are possible mechanisms involved.

Fig 3 shows a time instant of a spherical air cavity, travelling from inception in the minimum pressure region and growing to its maximum radius $r_{M}$, tack length $L_{M}$ and tack angle $\phi_{M}$, at the rupture point at the meniscus, with this process repeating continuously to induce a pressure wave in the far field. The wave equation will not be solved here and the presence of the meniscus is only treated as a limit to the pressure amplitude; nor are nonNewtonian rheology and viscoelasticity of the ink taken into account. As indicated by the nip clearance (twice the film thickness on the roller), the ink is assumed incompressible. The pressure gradient caused by compression of the resilient roller is implied, but not specifically shown, in Fig 3 .

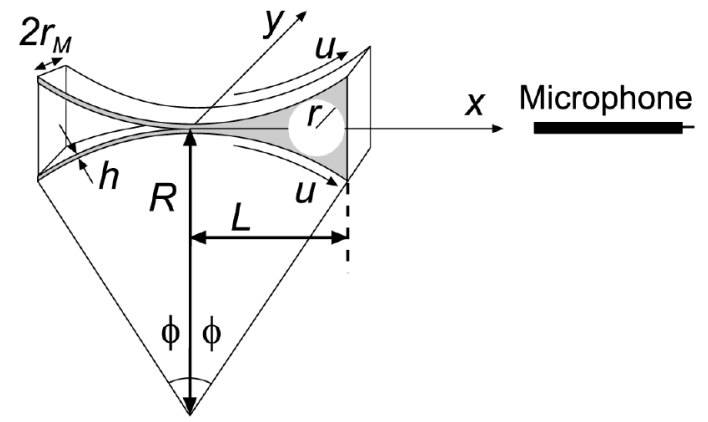

Fig 3. Nip entrance and exit, showing the simplified model for the latter, i.e. with single cavity having travelled from inception to this rupture point, inducing a pressure wave in the far field

In the simplified model, all travelling and rupturing cavities in the nip exit are then represented by a row (along the y-axis) of independent, single, time-averaged cavities. Accordingly, only the average power of the sound emission is described, not the frequency, which would require consideration of the discrete events. Some part of the air may accumulate to a constant level in the ink and some part will burst out and produce sound. To ensure a homogeneous ink distribution the ink mass flow rate is constant over the nip. Due to ink incompressibility, continuity and geometry then demand that:

$$
\begin{aligned}
& 2 \pi r^{3} / 3=r_{M} R^{2}(2 \sin \phi-\phi-\sin \phi \cos \phi) \\
& r=\left(\frac{r_{M} R^{2}}{2 \pi}\right)^{1 / 3}\left[\phi-\frac{7}{20} \phi^{3}+O\left(\phi^{5}\right)\right]
\end{aligned}
$$

If there is no mass transport over the ink-cavity boundary, and an infinite effective medium exists outside the cavity, mass conservation and integration of the Navier-Stokes equation leads to an inertia-dominated pressure $p$ in the far field from the cavity (Brennen 2005 pp. 101, 142):

$$
p=\frac{N_{c} \rho_{L}}{x} \frac{d}{d t}\left(r^{2} \frac{d r}{d t}\right)
$$

where $N_{c}$ is the effective number of such cavities along the $y$-axis that contribute to the total sound (also facto- 
ring in the microphone's lateral resolution) and $\rho_{L}$ is the density of the ink medium, taken to be Newtonian. The cavity expansion is assumed adiabatic, i.e. its volume expansion due to the under-pressure in the ink produces an equal negative pressure contribution to the cavity according to $E q 1$ until rupture. Since the energy balance is not considered in this geometrical mass-conservation model, heat dissipation is omitted, though it is allowed and accounts for part of the tack energy not becoming sound energy.

Since the angle $\phi$ is always small, the first non-zero term in the Taylor expansion of $E q 7$ suffices for substitution into $E q$ 8, to yield the maximum pressure emitted at film rupture

$$
p_{M} \approx \frac{N_{c} \rho_{L} u^{2}}{x}\left(\frac{2 r_{M}^{5}}{\pi^{2} R^{2}}\right)^{1 / 3}
$$

where $u=R \phi / t$ denotes the roller surface speed in Fig 3 . Accordingly, the pressure increases with the maximum cavity radius as might be expected. However, we will here eliminate $r_{M}$ from the expression since it is very difficult to measure, and instead use the advantage of the known ink film thickness, $h$, via the dimensionless quantity $\varepsilon$ that is defined as the ratio of volume fraction of air to ink in the nip at film rupture. Following $E q 7$ and Fig 3, this is given as:

$$
\varepsilon \equiv \frac{\pi r_{M}^{2} / 3}{R \phi_{M} h} \approx \frac{1}{h}\left(\frac{\pi^{2} r_{M}^{4}}{54 R}\right)^{1 / 3} \approx \frac{L_{M}^{2}}{6 h R}
$$

where $L_{M}=R \sin \phi_{M} \approx \mathrm{R} \phi_{M}$. After squaring according to Eq 3 and division by two assuming sine wave behaviour, Eq 9 becomes

$$
P_{a}=\frac{p_{M}^{2}}{2} \approx 0.7 \frac{N_{c}^{2} \rho_{L}^{2} u^{4}}{x^{2} R^{1 / 2}}(\varepsilon h)^{5 / 2}
$$

Since the film thickness is proportional to the ink load (in $\mathrm{g} / \mathrm{m}^{2}$ ), it follows from $E q 11$ that the average power $P_{a}$ is predicted to increase as the 2.5 power of ink load, provided $\varepsilon$ is assumed independent of this parameter. Thus, on substituting into $E q$ 5, the model then predicts that average power in $\mathrm{dB}$ is linearly related to $\log _{10} h$ with slope 25 . sound emitted from the nip exit at a load of $5 \mathrm{~g} / \mathrm{m}^{2}$ of the mineral oil-based offset ink. Observe that the power slowly increases over the first $30 \mathrm{~min}$., as a consequence of the partial drying of the ink increasing its tack, and thus the work performed during film splitting. The distribution of frequencies contributing to this average power (see $E q$ 3-4) is given by the power spectral density ( $P S D$ ) in Fig $4 b$, shown for a range of loads of this ink. At low frequencies, below around $1-2 \mathrm{kHz}$, all spectra display a common and apparently ink-independent contribution, arising from the inker motor and machinery, while at higher frequencies the ink splitting sound dominates and progressively increases with ink load in this interval, in each case displaying a local maximum around $35 \mathrm{kHz}$. For the two lowest amounts in Fig $4 b$ an extra spike occurs at approx. $10 \mathrm{kHz}$, arising from friction or slipstick of the roller surface, subsequently vanishing at higher loads due to the increased ink coverage and lubrication.

Fig $5 a$ and $b$ display the average power and average frequency, respectively, calculated via Eqs. 3 and 6 from the PSD's at shorter times, for the mineral oil-based ink at all studied loads in the range $0.5-100 \mathrm{~g} / \mathrm{m}^{2}$. In each case a high-pass filter has been used at $5 \mathrm{kHz}$, thus excluding the lower frequency machine contributions mentioned above. However, its is apparent from Fig $4 b$ that this filtration is not strictly necessary to distinguish the ink film splitting, which dominates this machinery sound, but was performed rather for smoothing reasons. Four regions with different functional dependence on ink load can be clearly defined in Fig 5. Region I shows an increase in power and frequency, followed by a more clearly linear power development (on this log-log plot, i.e. power law in a linear plot) in region II. Over region III the power continues to increase linearly, though with a lower slope, while the frequency now decreases. As even greater amounts of ink are added, in region IV, the average power decreases strongly (while frequency increases again), however this is due to a slipping contact between the friction-driven polymer roller and metal roller. These extreme loads in region IV greatly exceed those typically used in the ink train in sheet-fed offset printing, and thus have little relevance. The interpretation of behaviour in regions I-III of principle relevance is discussed below.

\section{Results and Discussion}

\section{Ink splitting between rollers}

We begin with the simpler case of film splitting in the ink distributor unit illustrated in Fig 1 a. In contrast to the laboratory printing of single strips of paper, the ink splitting on the continuously rotating inking unit is in principle time invariant, aside from slow ink drying effects. Thus its acoustic signal is effectively stationary and the data sampling has greater reproducibility. Fig $4 a$ plots the long-time evolution in average power of the
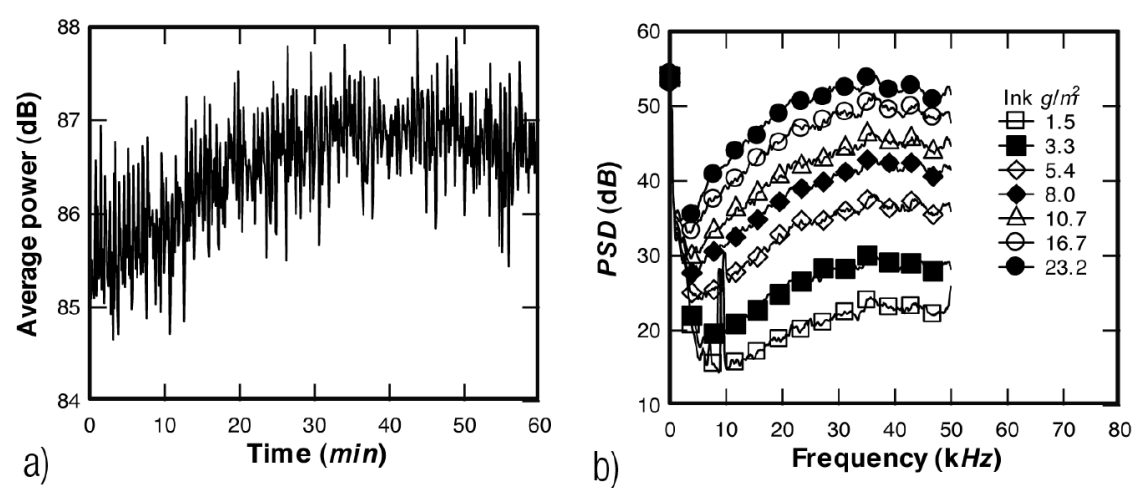

Fig 4. a) Evolution of average power over long times from the inking unit at constant ink amount $\left(5 \mathrm{~g} / \mathrm{m}^{2}\right)$. b) Power spectral density $(P S D)$ of successively higher ink loads. 

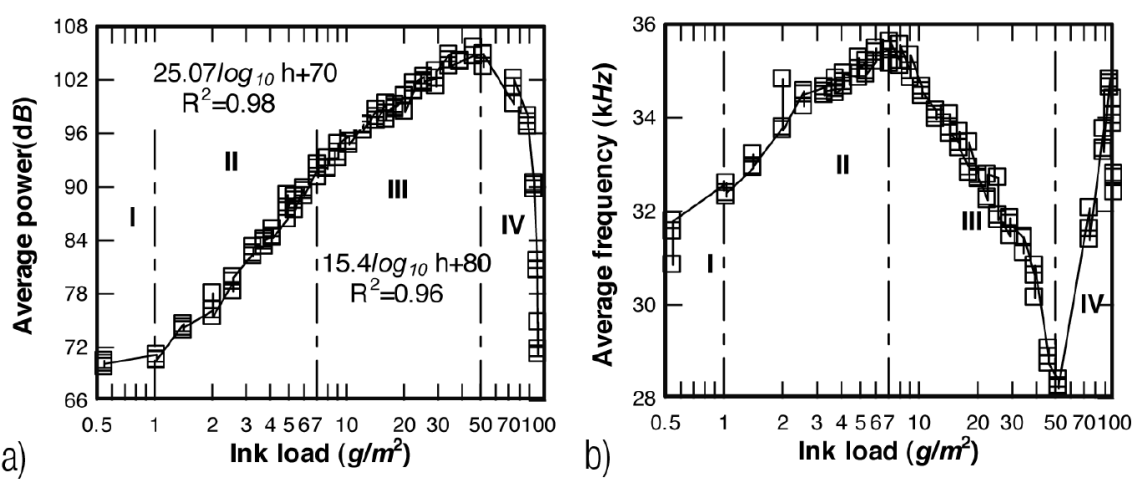

Fig 5. a) Average power, and b) average frequency, as a function of amount of the mineral oil-based ink on the inker, with a high pass filter applied at $5 \mathrm{kHz}$. The slope of 25.07 in region // of a) agrees with Eqs 11 and 5 with the film thickness, $h$, being proportional to the ink load.

To provide further insight into the nature of the sound emitted, the time to self-stop of the top roller after switching off the motor was used as an indirect indication of ink tack. The subsequent evolution of the average power (with the results smoothed and averaged from 10 replicates) is shown in Fig $6 a$ for a range of ink loads, with the origin $(0 \mathrm{~s})$ corresponding to the time of motor switch-off. This starting point was determined by lowpass filtering the signal to detect the button push-release sound followed by threshold triggering. At short times the average power increases with ink amount, as expected from Fig 5a, while at times approaching the stop point this simple hierarchy no longer applies. However, accurate determination of roller stop-time from average power is difficult, since the relative difference between samples decreases there. A somewhat more accurate method uses the average frequency, in Fig $6 b$, as it does not decrease in magnitude as long as the roller rotates. Fig $6 c$ shows the time to roller stop thus estimated from average frequ-


Fig 6. a) Average power, and b) average frequency, from the inking unit as function of time after motor switchoff, for a range of loads of the mineral oil-based ink. High pass filter applied at $5 \mathrm{kHz}$. c) Time to roller stop estimated from b). ency. The sensitivity of this indirect tack measurement is also limited by the high inertial force of the roller mass relative to tack force. Nevertheless, Fig $6 c$ displays a clear trend, the correlation of which to Fig $5 a$ is addressed below.

On addition of ink in region $I$ in Fig $5 a$ cavities are formed and expand in the nip, with both their energy and rate consequently increasing with ink amount. Over this region a full lubricating film is developed, however the resulting reduction in roller frictional noise is more than compensated for by the power increase from cavitation. Over region II the average power and frequency continue to rise, with the former following a power law with exponent from least squares fitting (see Fig $5 a$ ) very close to that of 2.5 , or a factor 25 on $\mathrm{dB}-\log$ scale as predicted in the scaling of $E q 11$. Thus, despite the simplifications made throughout the derivation of this model, its predicted exponent is consistent with these assumptions and with constancy of air/ink volume ratio $\varepsilon$ in this region, or in other words with tack length $L_{M}$ increasing as the square root of the film thickness ( $E q 10)$. Furthermore, substitutions of known parameter values reveal that the magnitude of the prediction in $E q 11$ is also in reasonable accord with measurements for sensible estimates of tack length and cavity number density $N_{c}$. The increasing frequency may be explained by an increasing number of cavitation events per unit time, resulting from filament splitting over this extended tack length from the nip, and an increasing size distribution of cavities due to increased likelihood of coalescence further from the nip. In this region II the time to roller stop in Fig $6 c$ increases to a maximum, with the decrease in hydrodynamic resistance consistent with expectations from lubrication theory (Smith 1997a), since the pressure gradient at the nip exit decreases with increasing ink film thickness.

Over region III the average power continues to exhibit a power law scaling, however now with the lower exponent of 1.5 , or a factor 15 on dBlog scale (see Fig 5a), coinciding with the sudden decrease in average frequency. To pinpoint the cause for the over-prediction of power from the model in this region III is challenging, since almost all of its assumptions err on this side of over-estimation. While it appears likely that the lower exponent in region $I$ is due to the ratio $\varepsilon$ decreasing with ink amount (to approach its stable value in region II), due to the extra ink load improving the sub-optimal coverage and air-seal in 
the nip, such an explanation cannot apply in region III. One factor possibly contributing to this loss of power and reduction in frequency is the observed onset of ribbing in region III, which could hinder the cavities from elongation or decrease their number density $N_{c}$ in this direction. In region III the time to roller stop now decreases with ink load, as would normally be expected of ink tack, presumably since the normal stress contribution from the filament elongation now dominates over the shear lubrication effect of the ink. Note that the ribbing seen in region III disappeared on further increase of ink load in region $\mathrm{IV}$, due to decrease in roller speed to below the critical value by virtue of the sliding of the frictiondriven top roller.

\section{Printing on coated paper}

An example of a transduced acoustic raw signal, i.e. pressure variation as a function of time, recorded during the printing of a single paper strip is displayed in Fig $7 a$. In order to isolate the relevant features, the average power was calculated from this, and is shown in Fig $7 b$. After high pass filtering at $5 \mathrm{kHz}$ (i.e. as performed above for the inking experiments) the average power in Fig $7 \mathrm{c}$ now reveals the known fact that the rotation of the cylinder over the recording time can be divided into three stages, where the second stage involves the contact between the print cylinder and the paper sample. The supposition that the extra sound from the printing region arises from ink film splitting, and not merely frictional effects, is justified by the power spectral density (PSD) curves in Fig $7 d$, displaying power increasing with ink load. Clearly, the
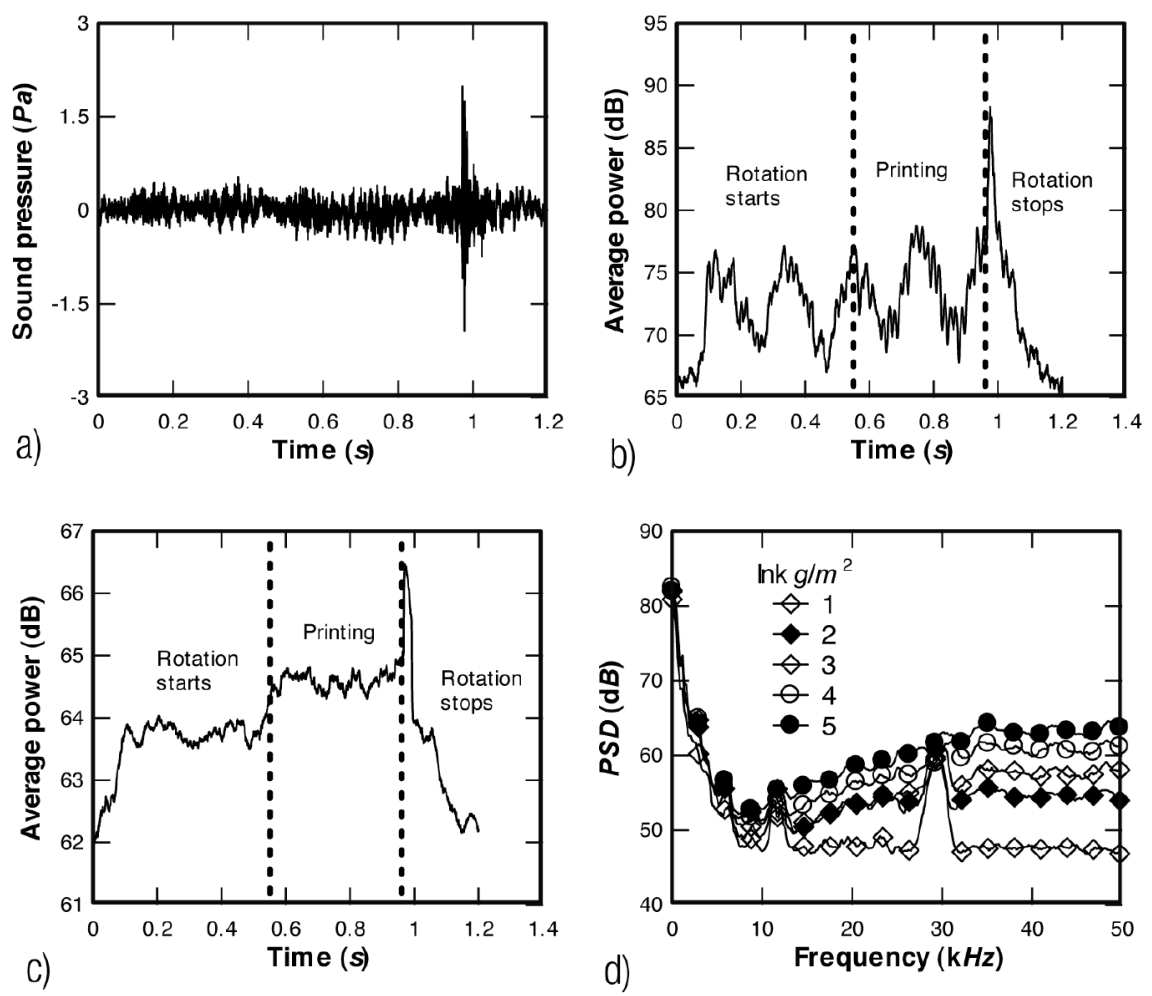

Fig 7. a) Sound pressure variation from the printing unit nip for $3 \mathrm{~g} / \mathrm{m}^{2}$ of the mineral oil-based ink applied to the glossy-coated paper; b) Average power obtained from a) without frequency filtering; c) Average power after high pass filtering at $5 \mathrm{kHz}$; d) Power spectral density (PSD) for the five different ink loads. Printing region signal in b) was used to calculate the $P S D$. main distinction between the samples is manifested above around $5 \mathrm{kHz}$, whereas the contributions at lower frequencies originate principally from the printer motor and machinery effects, and are thus relatively independent of ink amount. Note that the machinery contributions are much louder (and also extend up to somewhat higher frequencies) than was the case for the ink distributor in Fig 4b, and thus high pass filtering at around this value is now essential for extraction of the film splitting contribution. The maximum power is seen to lie outside the measured frequency range, implying that the aliasing described above may have influenced the spectrum, somewhat more than for the signal from the ink rollers.

Fig $8 a$ plots the average power for each of the five amounts of the two offset inks (mineral and vegetable oilbased) printed on the two papers (matte and glossy coated), evaluated using $E q 3$ and in each case averaged over the printed area (i.e. as opposed to over short sub-intervals as in Fig 7c to follow its evolution over such an area). Note that for each combination of ink amount and type and paper type, five replicate printings were performed, and indicated in Fig 8 by the curve linking these five identical symbols. The film-splitting power (averaged over these replicates) increases in all cases with ink amount, as for the ink distributing unit in Fig $5 a$. Although each curve can be reasonably well-fitted to a power law on linear scale, the exponents lie in the range $1-1.5$, i.e. substantially less than the value of 2.5 for equivalent ink amounts on the roller in Fig $5 a$ and predicted by theory. Significantly, the results exhibit clear and systematic differences between the ink-paper combinations, implying that the acoustic technique (and moreover the simplest first-order measure of average power obtained from it) is sufficiently sensitive to detect film splitting differences within the same class of commercial sheet-fed offset ink and coated fine paper. Note though that these distinctions are most apparent at ink loads in excess of usual levels in printing.

In particular, the glossy paper gives rise to higher power than its matte counterpart at all ink levels (aside possibly from the lowest) and for both ink types. This is in accordance with expectations, for which a number of mechanisms could contribute to this effect. For the rougher matte paper (see Table 1) the ink film in the nip is presumably less air-tight than for the glossy paper, or in the language of Eq 11, its ratio $\varepsilon$ decreases more strongly with thickness $h$. Related to this, the matte paper provides a greater number of asperities at which cavities can grow and thus rupture faster and with less energy. Further, its generally lower ink-paper contact area 

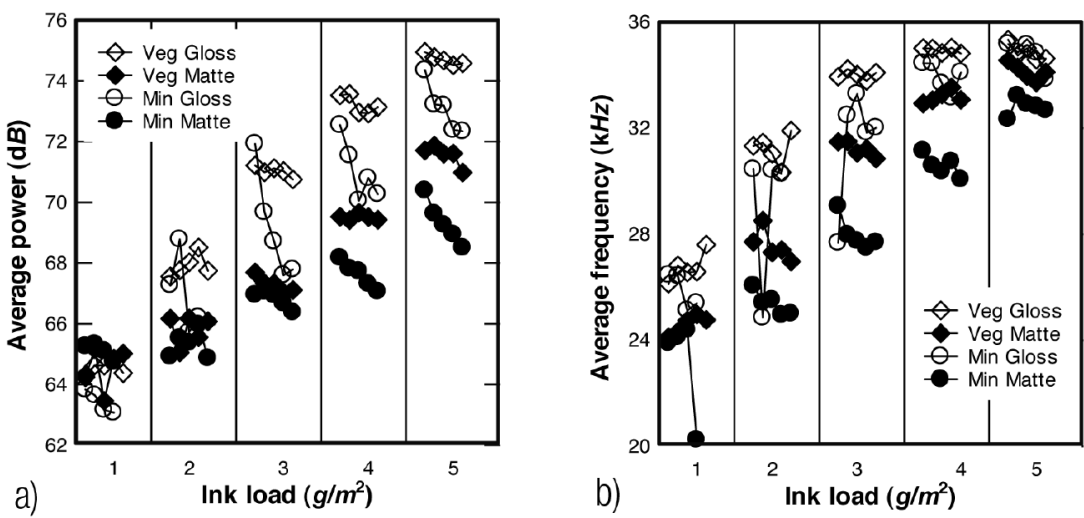

Fig 8. a) Average power, and b) average frequency, over the printing region (e.g. in Fig 7c) for the five loads of the two inks (mineral and vegetable oil-based) printed on the two papers (matte and glossy coated). High pass filtering was applied at $5 \mathrm{kHz}$. Vertical lines separate each series of connected points for which the print disc was inked from the same supply on the inker unit. Time between consecutive printing points is approx. 4 min. (including 1 min. redistribution on the roller, 1 min. inking of the print disc, and $2 \mathrm{~min}$. weighing and transport).

would be expected to reduce tack force per nominal area and thus also the corresponding acoustic power. It is also conceivable that the acoustic method detects the onset of ink setting (see below), known to occur more rapidly on the glossy paper by virtue of its finer surface pores, thus increasing the ink viscosity and hence the work of film splitting. Average power increases somewhat from mineral- to vegetable-oil based ink, for each paper and again at all ink amounts with the possible exception of the lowest. This trend may be directly due to higher ink tack, or also reflect differences in air contents of the inks in the nip, and is again discussed below.

Further, observe that the replicates exhibit a clear and systematic hysteresis (especially apparent at the three highest ink levels), i.e. for their consecutive inking and printing at a given ink level, the average power decreases in the running order from first to last replicate. Although this suggests that increased distribution time on the inking unit shifts the power on printing to lower values, the average power from the splitting on the inking unit itself displayed the opposite trend (see Fig 4a), namely increasing slowly over these time periods. The hysteresis effect in Fig $8 a$ is noticeably more pronounced for the

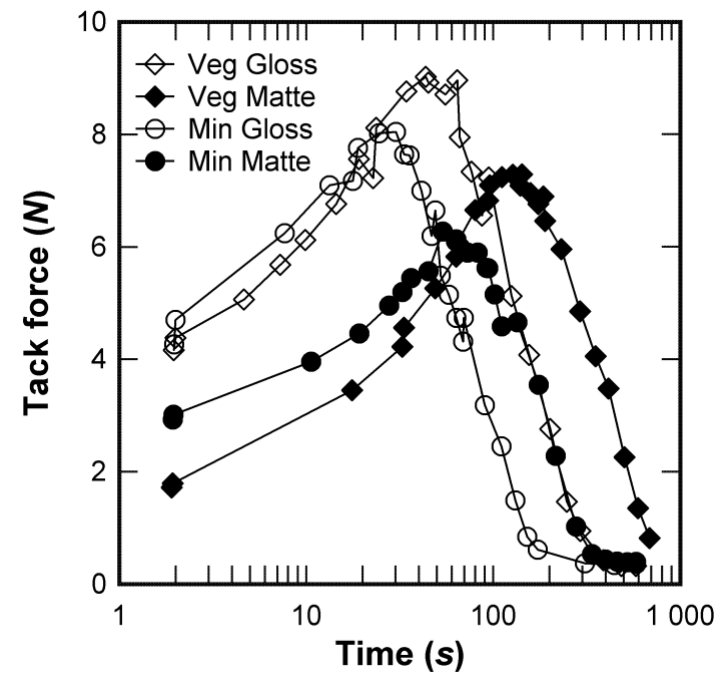

Fig 9. Tack force evolution from ISIT for the same four ink-paper combinations as in Fig. 8, now at a fixed ink load of $2 \mathrm{~g} / \mathrm{m}^{2}$, and with each point being the average of 9 replicates. The average errors for all curves are within the limit of $0.5 \mathrm{~N}$. mineral oil-based ink, with its vegetable oil counterpart displaying greater constancy. The corresponding average frequency curves in Fig $8 b$ display a similar general hierarchy, with glossy paper and/or vegetable oil giving higher frequency than matte paper and/or mineral oil. However note that average frequency appears more suitable a measure for distinguishing behaviour at lower ink amounts, i.e. the reverse of that for average power, possibly related to a similar slow approach with ink load to an insensitive maximum in average frequency as exhibited on the inker unit in Fig $5 b$.

Fig 9 shows the pull-off tack force development as measured by ISIT (see Fig 1) for the same inks and papers as in Fig 8. Since the force is measured after printing, the first point at $2 \mathrm{~s}$ is still not early enough to be directly comparable (in terms of vehicle concentration) to the sound recorded during printing, and is subject to significant measurement uncertainty if not supported by results from subsequent pull-offs. In this sense the maximum tack value measured, or the slope of tack build-up to this maximum, provides a more reliable quantity. Note that the maximum tack increases in the same order as the average power in Fig 8 a, i.e. from mineral (oil-based ink) on matte (paper) to vegetable on matte to mineral on glossy to vegetable on glossy. This could be interpreted as an indication that setting already influences tack force and average power in the nip exit, however as noted above, other mechanisms related to cavitation (i.e. more directly relevant to nip behaviour) would also predict higher average power on the glossy paper.

\section{Conclusions}

This investigation reinforces previous observations that ink film splitting noise, as measured in the vicinity of laboratory nips, is a broadband high frequency sound, moreover showing remarkable sensitivity to ink load and characteristics (i.e. vegetable or mineral oil-based sheetfed offset inks) and paper (i.e. variation of roughness of fine coated papers). In the simplistic model suggested here, assuming spherical expanding cavities in a symmetric rigid nip with a Newtonian ink, the average power is predicted to increase as the 2.5 power of ink load. This prediction holds experimentally on an IGT inking unit for intermediate ink loads $\left(1-7 \mathrm{~g} / \mathrm{m}^{2}\right)$ whereas overestimation appears at lower and higher loads, presumably owing to ink sparseness (i.e. lack of ink for volume expansion) and ink flooding (i.e. ribbing instability of cavities), respectively.

Indirect determination of ink tack through time to inker roller stop after motor switch-off reflects that cavitation, in the intermediate ink load region, promotes the loss of ink film resistance along with the lower hydrodynamic resistance as predicted by lubrication theory, pro- 
bably valid for this relatively low speed, at increasing ink loads. At higher ink loads, however, increasing extensional forces and less cavitation due to ribbing may explain the increase in tack there. For the printing unit experiments, the theory again overestimated the power-law dependence of acoustic average power on ink amount, with the measurements also revealing a clear dependence on paper type, with the glossy paper producing higher power than its matte counterpart. This deviation of theory from experimental observation is thought to be primarily due to the influence of paper roughness on air flow and cavitation, analogous to the sparseness on the ink distributor, although the trends are perhaps misleadingly similar to those expected from consideration of ink setting.

\section{Acknowledgement}

The work was performed as a part of the PrintTech Research (T2F) Program. We thank the Foundation for Knowledge and Competence Development (KK stiftelsen), The Swedish Pulp and Paper Research Foundation and Stiftelsen Grafsk Forskning for partly financing this work. All gratitude to Mikael Sundin at YKI for performing the ISIT ink tack measurements.

\section{Literature}

Amari, T., Xiamfu, W., Hayashi, T. and Morita, K. (1994): Dynamics and rheology of inks including various types of gelling agents, In: TAGA Proc.

Banks, W. H. and Mill, C. C. (1953): Tacky adhesion-a preliminary study, J. Colloid Sci. 8, 137.

Bery, Y. A. and Loel, P. A. (1992): Nature of film splitting, In International Printing and Graphic and Graphic Arts Conference, TAPPI, 53-74.

Bikerman, J. J. (1968): The science of adhesive joints, Academic Press.

Blake, J. R. and Gibson, D. C. (1987): Cavitation bubbles Near Boundaries, Annu. Rev. Fluid Mech. 19, 99-123.

Blake, W. K. (1986): Mechanics of flow-induced sound and vibration, Academic Press.

Brennen, C. E. (2005): Fundamentals of Multiphase Flows, Cambridge University Press.

Carvalho, M. S. and Scriven, L. E. (1996): Capillary and viscoelastic effect on elastohydrodynamic lubrication in roller nips, J. Tribology, 118, 872-879.

Ceccio, S. L. (1990): Observation of the dynamics and acoustics of traveling bubble cavitation, California Institute of Technology, Diss.

Cioc, S. (2004): Application of the space-time conservation element and solution element numerical method to flows in fluid films, University of Toledo, Diss.

Coveney, V. A. (1978): Cavitation between immersed rollers, Watford College, Diss.

Coyle, D. J. (1988): Forward roll coating with deformable rolls: A simple onedimensional elastohydrodynamic model, Chem. Eng. Sci. 43(10), 2673-2684.

De Grace, J. H. and Mangin, P. J. (1984): A mechanistic approach to ink transfer. Part I, Effects on substrate properties and press conditions.

De Grace, Y. H., Dalphond, J. E. and Mangin, P. J. (1992): A Mechanistic Approach to Ink Transfer, Part III: Properties of Ink Filaments in Printing Nips, In Advances in Printing Science and Technology, 21, 312.

Dowson, D. and Higginsson, G. R. (1966): Elasto-Hydrodynamic Lubrication, Oxford Pergamon Press.

Dowson, D. and Taylor, C. M. (1979): Cavitation in bearings, Annu. Rev. Fluid Mech. 11, 35-66.
Ercan, S. N. (2001): The influence of process parameters on filament size distribution, University of Maine, Diss.

Fahy, F. (2003): Foundations of Engineering Acoustics, Academic Press.

Gane, P. A. C. and Seyler, E. N. (1994): Tack development: An analysis of ink/paper interaction in offset printing, In TAPPI, Coating Conference.

Glatter, T. and Bousfield, D. (1996): Print Gloss Development on a Model Substrate, In International Printing and Graphic Arts Conference, 141.

Hayashi, T. and Amari, T. (1992): Dynamics of transfer and splitting of emulsified ink, In International Printing and Graphic Arts Conference.

Hayashi, T., Morita, K. and Amari, T. (1993): Rheological properties and printabilities of Polybutadiene/Carbon Black Ink, J. Jpn. Soc. Colour. Mater. 66(11), 655-644.

Holroyd, T. J. (1997): Acoustic emission- an NDT technique evolving into a versatile industrial monitoring method, Measurement and Control, 30(5), 141-145. Iwasaki, Y., Takahashi, Y., Yukawa, H. and Kada, H. (1993): Study on paper releasing phenomena in offset printing by an acoustic approach, In TAGA Proc. 238.

Lim, C. H., Bohan, M. F. J., Claypol, T. C. and Getin, D. T. (1996): A finite element investigation into a soft rolling contact supplied by a non-newtonian ink, J. Phys. D: Appl. Phys. 29, 1894-1903.

Macphee, J., Shieh, J. and Hamrock, B. J. (1992): The application of elastohydrodynamic lubrication theory to the prediction of conditions existing in lithographic printing press roller nips, In Advances in Printing Science Technology, IARIGAI, 242-276.

Myers, R. R., Miller, J. C. and Zettlemoyer, A. C. (1958): The splitting of thin liquid films. Kinematics, J. Colloid Sci. 14, 287-299.

Oppenheim, A. V. and Willsky, A. S. (1997): Signals and Systems, Prentice Hall Int. Inc.

Patel, N. and Dealy, J. M. (1987): Measurement of tensile stress at the exit of a printing nip, J. Non-Newtonian Fluid Mech. 22, 245-252.

Pearson, J. R. A. (1960): The stability of uniform viscous flow under rollers and spreaders, J. Fluid Mech. 7, 481-500.

Press, W. H., Flannery, B. P., Teukolsky, S. A. and Vetterling, W. T. (1992):

Numerical recipes in C, Cambridge University Press.

Sjodahl, L. H. (1951): Ink flow on rotating rollers, Am Ink Maker, 3, 31.

Smith, M. K. (1997a): Asymptotic methods for the mathematical analysis of coating flows, In Book Coating flows Edited Smith, M. K., Chapman and Hall, 263.

Smith, S. W. (1997b): The scientist and engineer's guide to digital signal processing, California Technical Publishing.

Taylor, J. H. and Zettlemoyer, A. C. (1958): Hypothesis on the mechanism of ink splitting during printing, Tappi, 41(12), 749.

Thomson, I. G. and Young, F. R. (1975): High-speed photographic studies of ink filamentation, J. Oil Colour Chem. Assoc. 58, 389.

Welch, P. D. (1967): The use of Fast-Fourier Transform for Estimation of Power Spectra: A Method Based on Time Averaging Over Short, Modified Periodograms, Audio Electroacoustics, 15, 70-73.

Williams, J. E. F. (1969): Hydrodynamic noise, Annu. Rev. Fluid Mech. 1, 197-222. Voet, A. and Geffken, C. F. (1951): The nature of tack, Ind. Eng. Sci. 43, 16141623.

Voltaire, J. (2004): Ink film splitting acoustics and tack on paper in offset printing: A laboratory and theoretical study, Royal Institute of Technology, KTH, and Institute for Surface Chemistry, YKI, Lic.

Voltaire, J., Mattila, U., Fogden, A., Nieminen, S. and Reinius, H. (2004): Acoustic charachterisation of film splitting in a HSWO printing nip, In: International Printing Graphic Arts Conf. 1, 21.

Young, F. R. (1999): Caviation, Imperial College Press.

Manuscript received December 30, 2005 Accepted May, 2006 